\title{
Development of an Index for Drop-Foot Severity of DPN Patients
}

\author{
Albert K. Chong1, Suhad K. R. Al-Magsoosi1,2* \\ ${ }^{1}$ Faculty of Health, Engineering and Sciences, University of Southern Queensland, Toowoomba QLD, Australia \\ ${ }^{2}$ University of Wasit, Kut, Iraq \\ Email: Chonga@usq.edu.au, ^U1052339@umail.usq.edu.au
}

How to cite this paper: Chong, A.K. and Al-Magsoosi, S.K.R. (2019) Development of an Index for Drop-Foot Severity of DPN Patients. Journal of Biosciences and Medicines, 7, 61-64.

https://doi.org/10.4236/jbm.2019.75009

Received: March 23, 2019

Accepted: May 14, 2019

Published: May 17, 2019

\begin{abstract}
Currently, the population percentage of diabetics suffering Diabetic Peripheral Neuropathy (DPN) and foot-drop gait anomaly was estimates as 15\% in the United States. The onset of foot-drop could not be detected until symptoms could be observed visually, patient falling or patient experiencing painful gait issues and expensive medical tests. This research showed that by utilizing the plantar-pressure characteristics of DPN drop-foot gait, a set of index could be developed for the severity of DPN.
\end{abstract}

\section{Keywords}

Plantar Pressure Sensor, Gait, Foot-Drop, DPN, Diabetic Index

\section{Introduction}

Recent studies [1] highlighted the total numbers of more than 1.9 billion adults, 18 years and older, were overweight which included 650 million classified as obese [2]. The increase in the number of Type 2 diabetes (T2DM) was associated with the raise in overweight and obesity. Authors in [3] argued that of the two hundred participants with T2DM participated in the study, ninety-one per cent of participants were either overweight or obese. This rate becomes a major public health concern worldwide as it is often associated with both increasing health care cost and disability [2] [4]. These authors reported seventy-six per cent reported altered sensation in their lower limbs. Only $22.2 \%$ of participants reported having examined their feet and gait characteristics and only when they experienced a problem with pain and gait issues. Drop-foot issue can often cause falling of older T2DPN sufferers [5] [6]. The purpose of the study is to assess whether drop-foot characteristics of T2DPN patient could be indexed based on 
gait plantar pressure characteristics which can be carried out at rural clinics at low cost.

\section{Methods}

\subsection{Plantar Pressure Sensors and Measures}

In-sole sensor (Figure 1) and F-Scan software (Version 6.70-03), Tekscan, Boston were used in the research. The measures of plantar pressure were peak pressure, pressure time integral, and pressure contact area over ten regions of the plantar surface, namely: (heel $(\mathrm{H})$, midfoot (MF), first metatarsal head (1 MH), second metatarsal head $(2 \mathrm{MH})$, third metatarsal head $(3 \mathrm{MH})$, fourth metatarsal head (4 MH), five metatarsal head (5 MH), hallux (1stT), second toe (2ndT), and three to five toes (3rd-5thT) (Figure 1 right) [1].

\subsection{Recruitment and Protocol}

In 2017, the investigation was approved by the Research Ethics Committee (proposal number: (H18REA065). All participants signed an informed consent form to participate voluntarily in the study [1].

The recruitment required older diabetic adults who were confirmed to have diabetic peripheral neuropathy (DPN) at two local hospitals in Malaysia. Also, older diabetic adults who have no DPN were recruited. Soft targets were placed on the plantar surface at the heel and at the second metatarsal areas for precise linear measurement of the foot on the pressure graph. A set of prototype stencils were used to position the targets. Subjects wore shoes with in-sole pressure sensors (TekScan) and walked on a straight line for roughly 20 steps and repeated six times. Based on the heel target position, the plantar distance covered from heel-down along the foot-path for a specific period of time was calculated and was called Plantar distance at the heel (PDH). Then, the amount of time it took to cover a quarter of the foot-path from heel-down and the amount of time it took to cover the foot-path from the second metatarsal head to toe-off were called Time at heel (TAH) and Time at push-off (TAP) respectively.

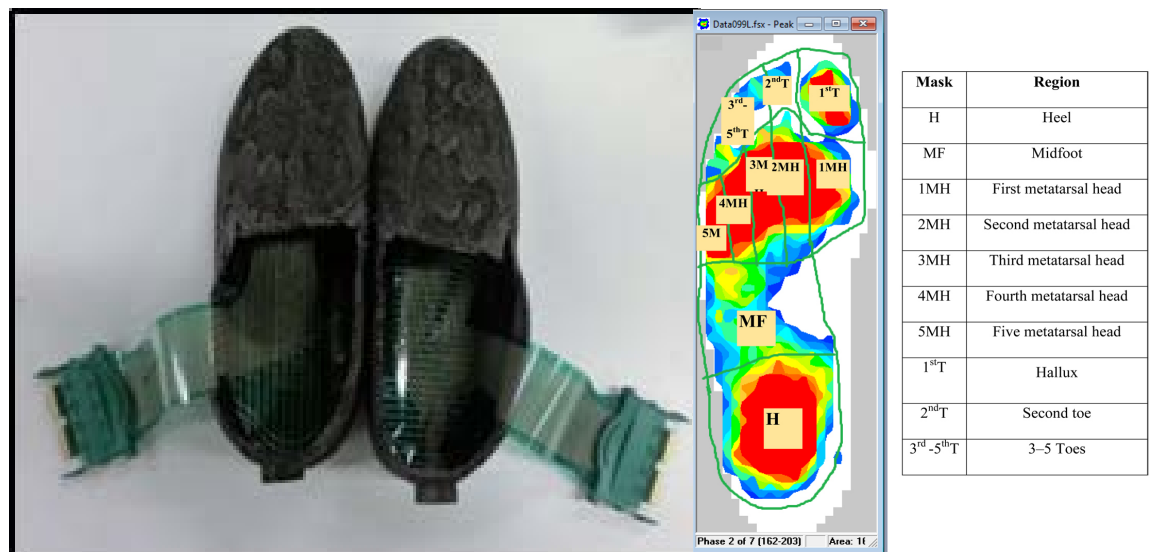

Figure 1. Foot insole sensorpad (left); Foot pressure sampling location (right). 
Table 1. New parameters derived from plantar pressure for drop-off severity indexing.

\begin{tabular}{cccccccc}
\hline $\begin{array}{c}\text { Non-DPN } \\
\text { Subject }\end{array}$ & PDH & TAH & TAP & $\begin{array}{c}\text { DPN } \\
\text { Subject }\end{array}$ & PDH & TAH & TAP \\
\hline 1 & 0.15 & 0.24 & 0.53 & 1 & 0.84 & 0.26 & 0.54 \\
2 & 0.37 & 0.29 & 0.42 & 2 & 0.78 & 0.16 & 0.65 \\
3 & 0.32 & 0.51 & 0.27 & 3 & 0.60 & 0.11 & 0.59 \\
4 & 0.30 & 0.27 & 0.52 & 4 & 0.88 & 0.25 & 0.43 \\
5 & 0.55 & 0.25 & 0.55 & 5 & 0.68 & 0.12 & 0.14 \\
6 & 0.34 & 0.32 & 0.52 & 6 & 0.95 & 0.09 & 0.41 \\
7 & 0.33 & 0.13 & 0.51 & 7 & 0.75 & 0.25 & 0.39 \\
8 & 0.38 & 0.21 & 0.57 & 8 & 0.96 & 0.09 & 0.40 \\
\hline
\end{tabular}

\section{Results}

The research results are presented in Table 1 . The PDH, TAH and TAP measures of the Non-DPN subjects and DPN subject are listed side by side.

\section{Discussion}

The PDH measures of the DPN subjects were significantly higher than the measure of non-DPN subjects. Also, the TAP measures were significantly higher in the former. Based on these measures it would be possible to calculate an index for foot-drop characteristics.

\section{Conclusion}

With the discovery of these measures, drop-foot anomaly could be determined at an early stage, thus DPN patients could have access to orthotics promptly, hence avoiding falling and other adverse effects such as plantar ulcer development, reluctance to walk and lack of enthusiasm for physical exercise. More subjects are required to determine the index values.

\section{Acknowledgements}

The first author thanks the staff (Professor Winnie Chee and Professor Verna Lee) and students at the International Medical University, Kuala Lumpur for their assistance. Without their enthusiastic help, this project would not have been successful.

\section{Conflicts of Interest}

The authors declare no conflicts of interest regarding the publication of this paper.

\section{References}

[1] Al-Magsoosil, S.K.R. and Chong, A.K. (2019) Foot Loading Pattern Variations between Normal Weights, Overweight, and Obese Adults Aged 24 to 50 Years. The 
2nd International Conference on Nursing and Healthcare (ICNH 2019), Kunming, May 29-31 2019.

[2] Sheffler, L.R., Bailey, S.N., Gunzler, D. and Chae, J. (2014) Effect of Body Mass Index on Hemiparetic Gait. $P M \& R, 6,908-913$. https://doi.org/10.1016/j.pmrj.2014.03.012

[3] Goie, T.T. and Naidoo, M. (2016) Awareness of Diabetic Foot Disease amongst Patients with Type 2 Diabetes Mellitus Attending the Chronic Outpatients Department at a Regional Hospital in Durban, South Africa. Afr J Prim Health Care Fam Med., 8, e1-e8. https://doi.org/10.4102/phcfm.v8i1.1170

[4] Anandacoomarasamy, A., Caterson, I., Sambrook, P., Fransen, M. and March, L. (2007) The Impact of Obesity on the Musculoskeletal System. Internal Medicine Journal, 37, A35.

[5] Swigchem, R., Roerdink, M., Weerdesteyn, V., Geurts, A.C. and Daffertshofer, A. (2014) The Capacity to Restore Steady Gait after a Step Modification Is Reduced in People with Poststroke Foot Drop Using an Ankle-Foot Orthosis. Physical Therapy, 94, 654-663. https://doi.org/10.2522/ptj.20130108

[6] Rossouw, H.A., Grant, C.C. and Viljoen, M. (2012) Overweight and Obesity in Children and Adolescents: The South African Problem. South African Journal of Science, 108, 1-7. https://doi.org/10.4102/sajs.v108i5/6.907 